\title{
A JANUS-PAPER PDMS PLATFORM FOR LAB-ON-A-CHIP APPLICATIONS
}

\author{
Rahim Rahimi ${ }^{l}$, Manuel Ochoa ${ }^{l}$, Tejasvi Parupudi ${ }^{l}$, Amy Donaldson ${ }^{3}$ \\ Mehmet R.Dokmeci ${ }^{2}$, Ali Khademhosseini ${ }^{2}$, Amir Ghaemmaghami ${ }^{3}$ and Babak Ziaie ${ }^{2}$ \\ ${ }^{1}$ Purdue University, West Lafayette, Indiana, USA \\ ${ }^{2}$ Harvard-MIT Health Sciences and Technology, Cambridge, MA, USA \\ ${ }^{3}$ The University of Nottingham, Nottingham NG7 2UH, UK
}

\begin{abstract}
A commercially available Janus paper with one hydrophobic (polyethylene-coated) face and a hygroscopic/hydrophilic one is irreversibly bonded to a PDMS substrate incorporating microfluidic channels via corona discharge surface treatment. The bond strength between the polymer coated side and PDMS is characterized as a function of corona treatment time and annealing temperature/time. A maximum strength of $392 \mathrm{kPa}$ is obtained with a $2 \mathrm{~min}$ corona treatment followed by $60 \mathrm{~min}$ of annealing at $120^{\circ} \mathrm{C}$. The water contact angle of the corona-treated polymer side decreases with increased discharge duration from $98^{\circ}$ to $22^{\circ}$. The hygroscopic/hydrophilic side is coated with hydrogel to show its potential for nutrient and chemical delivery in cell culture applications.
\end{abstract}

\section{INTRODUCTION}

There is a plethora of structural materials at the disposal of the microfluidic and lab-on-a-chip community, with PDMS and glass being the most popular and widely used ones. More recently, paper [1], [2] has garnered significant attention as a low-cost disposable platform for chemical [3]-[6] and biological assays. [5], [7] Paper-based devices are often fabricated either by using a hydrophilic paper that is patterned and impregnated with wax to create hydrophobic regions [8], [9] or alternatively by lasertreating a hydrophobic paper to create hydrophilic regions [10], [11]. In both cases, the hydrophilic/hygroscopic pattern is then used to perform colorimetric or electrochemical analyses on aqueous samples. In this manuscript, we introduce a unique and commercially available paper (Janus paper) with one hydrophobic (polyethylene-coated) face and a hygroscopic/hydrophilic one as a substrate for microfluidic and lab-on-a-chip applications. Such Janus papers can open doors to the development of microsystems with enhanced functionality and improved biomaterial integration capabilities. This is particularly true if it can be easily coupled with traditional microfluidics materials and systems.[12]-[14]

A paper exhibiting a Janus property is the freezer paper used to store frozen food. It is composed of a cellulose fiber mesh coated with polyethylene on only one side, thus being hydrophobic on the polymer side and hydrophilic/hygroscopic on the other side. At its hydrophobic face, such Janus paper bonds irreversibly to a polydimethylsiloxane (PDMS) microfluidic networks, enabling the fabrication of PDMS-paper platforms with dual surface energy; meanwhile, the hydrophilic/hygroscopic side is capable of supporting materials compatible with many paper processing techniques (e.g., chemical impregnation, gel coating, cell seeding).

Our experiments show that the polymer coating on freezer paper becomes hydrophilic upon exposure to a corona discharge/plasma. As a result, bonding to freezer paper can be accomplished without the need for additional adhesives, but rather via plasma-induced surface activation followed by thermal annealing (a technique typically used for bonding PDMS to PDMS or PDMS to glass [15]), thus enabling convenient integration of the Janus-paper platform with traditional microfluidic systems.
We implemented various standard microfluidic designs as a proof-of-concept demonstration. Traditionally, these systems are fabricated via a lithography/replica molding [12], [16] technique that requires (expensive) clean room facilities and multiple processing steps. We opted for a less expensive and more straightforward approach by defining channels on a PDMS substrate via a single-step laser ablation method and subsequently bonding them to the Janus paper.

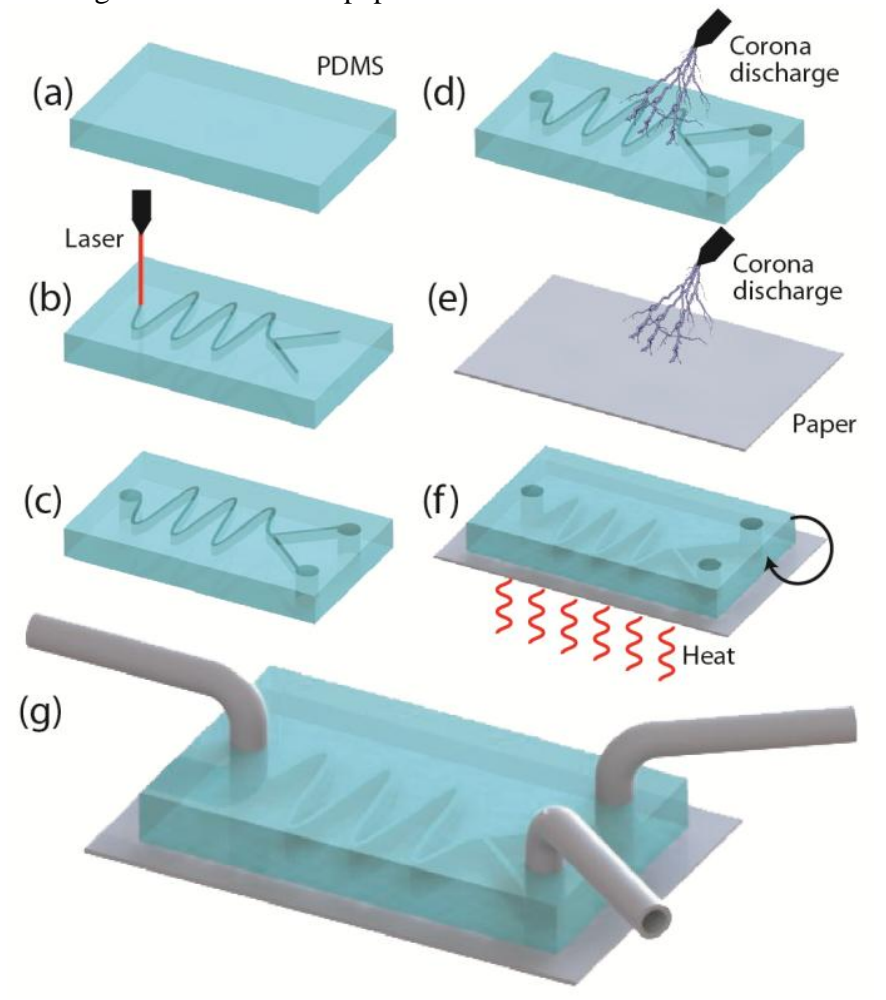

Figure 1: Conceptual illustration of the fabrication process. (a) Cast, crosslink, and release a PDMS substrate. (b) Laser-define the microfluidic network. (c) Punch inlet and outlet ports. (d) Treat the surface of PDMS and (e) the freezer paper with corona discharge or plasma. (f) Flip the PDMS and bring it into contact with the treated paper; press and anneal to bond. $(g)$ Attach tubing to microfluidic ports.

\section{DESIGN AND CHARACTERIZATION}

Figure 1 illustrates the fabrication technique for creating PDMS/Janus-paper devices. First, a $\mathrm{CO}_{2}$ laser engraver system (Universal Laser Systems Inc., Scottsdale, AZ) is used to directly write microchannel patterns on a substrate of crosslinked PDMS (Dow Corning Sylgard ${ }^{\circledR} 184,10: 1$ ratio, cured at $60{ }^{\circ} \mathrm{C}$ for $5 \mathrm{~h}$ ) by surface ablation (power $15 \mathrm{~W}$, speed $4 \mathrm{~mm} / \mathrm{ms}$ ). The resulting 
channels have a Gaussian cross-section $(200 \mu \mathrm{m}$ wide, $100 \mu \mathrm{m}$ deep); the shape can be further modified (e.g., rounder or deeper) by using an unfocused laser beam [17], [18] or multiple passes of the beam. After defining the channels on the PDMS, two $800 \mu \mathrm{m}-$ diameter holes are punched out to create inlet and outlet ports. Next, the PDMS and Janus paper (Freezer Paper, Reynolds Consumer Products, Inc.) are exposed to corona discharge using a hand held corona discharge unit (BD-10A High Frequency Generator, Electro-Technic Products, Inc.), and the two surfaces are subsequently brought into contact to create a permanent bond. The device is then annealed on a hotplate. Finally, $800 \mu \mathrm{m}-$ diameter pieces of Tygon ${ }^{\circledR}$ tubing are attached to the inlet and outlet ports.

Three major parameters that are known to affect the bond quality of PDMS to PDMS or PDMS to glass are the duration of corona treatment, the annealing temperature, and the annealing time. If the bonding process for PDMS-Janus-paper mimics that of the aforementioned ones, then similar trends can be expected for this structure. In particular, the surface energy of the polymer coating on the Janus paper is expected to decrease with increasing corona discharge exposure time, in turn increasing the affinity of one surface to the other. Higher annealing temperatures and extended annealing times can also increase the bond strength by relaxing the polymer on the paper and a allowing more intimate contact between the two bonding surfaces. To create strongly bonded PDMS-Janus-paper devices, we investigated the effect of these parameters on the overall bond strength.
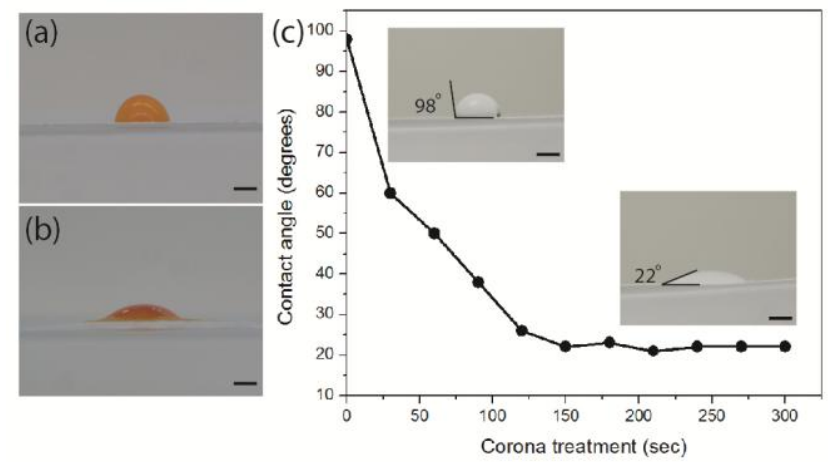

Figure 2: Surface properties of Janus freezer paper. Dyed agarose gel on (a) the hydrophobic (polymer-coated) and (b) the hydrophilic side of the paper. (c) Contact angle of a water droplet on the hydrophobic surface of freezer paper as a function of corona treatment duration. All droplet have a $4 \mu \mathrm{L}$ volume. All scale bars: $1 \mathrm{~mm}$.

A goniometry test setup was used to characterize the effect of corona discharge on the surface energy of the Janus paper (polymer-coated side only). The paper was exposed to corona discharge for various durations $(0-300 \mathrm{~s})$ and the water contact angle was subsequently measured using a $4 \mu \mathrm{L}$ droplet. The contact angle is plotted in Figure 2 as a function of exposure time. As is the case with PDMS, the corona treatment causes a significant reduction in angle (from $98^{\circ}$ to $22^{\circ}$ ).

This decrease can be attributed to the generation of oxygen polar groups on the polyethylene surface by plasma exposure [19], [20]. We observed that hydrophilicity of the polymer surface of the paper increases with plasma exposure time but saturates after 2 minute; therefore, this exposure time was used for all the PDMSpaper structures discussed in this manuscript.

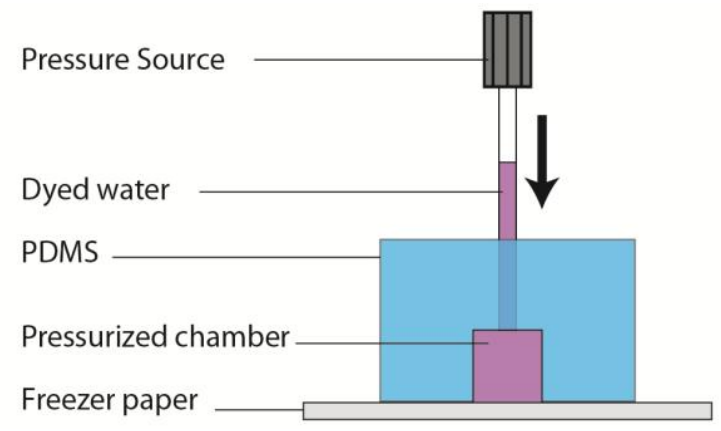

Figure 3: Test setup for measuring bond strength between freezer paper and PDMS. A gas source with a pressure gauge provided the pressure.

To characterize the maximum strength of the PDMS/Januspaper bond as a function of the annealing time and temperature, we fabricated multiple test devices with the design illustrated in Figure 3 . The structure consisted of a cylindrical chamber in a block of PDMS bonded to a Janus paper substrate. The PDMS block $(15 \mathrm{~mm} \times 15 \mathrm{~mm} \times 5 \mathrm{~mm})$ was formed from a master mold consisting of a laser-cut acrylic cylinder $(5 \mathrm{~mm}$ diameter $\times 2 \mathrm{~mm}$ height) adhered onto a petri dish with UV-curable adhesive (Loctite ${ }^{\circledR} 3105$ ). Next a $800 \mu$ m-diameter inlet port was cored out of the center of the PDMS block to create an inlet for the pressuring gas during the bonding test. A sheet of freezer paper was cut to match the dimensions of the PDMS mold. The PDMS and paper were then bonded together via corona discharge as previously described.

The ideal combination of annealing time and temperature required for a strong bond was determined by measuring the bond strength of the PDMS-paper structure created with various annealing temperatures $\left(40-140^{\circ} \mathrm{C}\right)$ and durations $(0-180 \mathrm{~min})$. To assess the quality of the bonding between the paper and the PDMS, a $800 \mu \mathrm{m}$-diameter syringe needle was inserted into the inlet port of the PDMS block and its perimeter was sealed with a polyurethanebased adhesive (Gorilla GlueTM, Gorilla Glue Inc.). The needle was connected to a syringe that was filled with dyed water to reveal potential leakages or device failure during the high pressure measurements. The open end of the syringe was connected to a pressure gauge and a flow-regulated nitrogen source in order to control the pressure in the PDMS/paper chamber. The pressure was gradually increased at a rate of $250 \mathrm{~Pa} / \mathrm{s}$ until the first sign of fluid leakage. Bonding failure was determined either by delamination of the two layers or by paper failure (tearing at the interface).

The pressure test data are plotted in Figure 4. The measurements show that low temperatures and short annealing times result in weaker bonds; in such cases, device failure occurs at the paper/PDMS interface. By increasing the annealing temperature and time the bond strength also improves up to 392 $\mathrm{kPa}$. This is comparable to bond strengths achieved via traditional oxygen plasma bonding and adhesive tape-PDMS techniques [21], [22]. The bond becomes strong enough that device failure occurs as a tear in the surface of the paper (rather than at the bonding interface), due to limitations in the paper mechanical strength of the paper. The data reveal an optimal annealing temperature and time of $120^{\circ} \mathrm{C}$ and 60 minutes for creating a strong irreversible bond between the paper and the PDMS. 


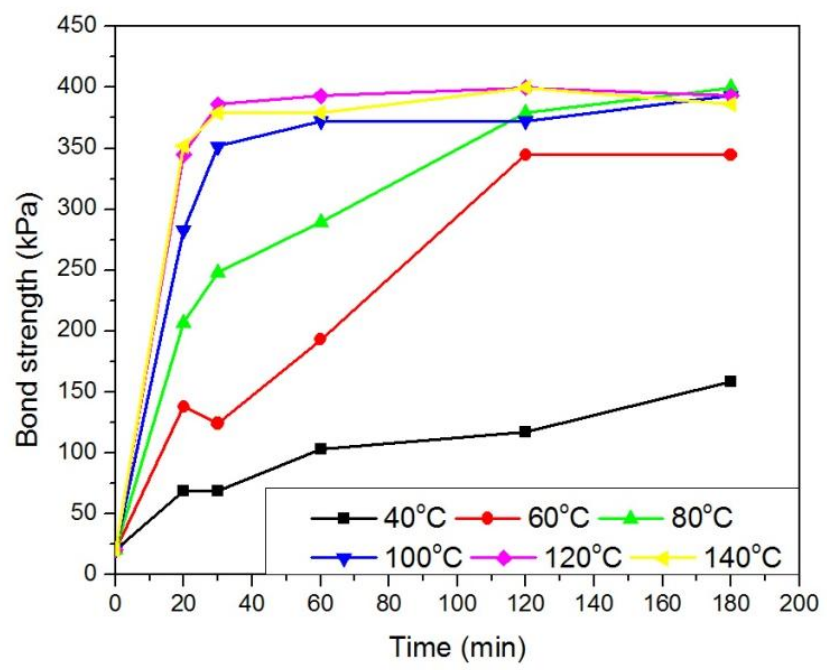

Figure 4: Bond strength as a function of annealing temperature and annealing. Note that at temperatures above $100^{\circ} \mathrm{C}$ annealing times beyond 20 minutes do not increase the bond strength significantly. Maximum strength occurs within 60 min at $T>80^{\circ} \mathrm{C}$ (for $T>100^{\circ} \mathrm{C} 20 \mathrm{~min}$ annealing time is adequate).

The optimal fabrication parameters obtained from the characterization experiments were implemented in the fabrication of PDMS-paper prototypes. Figure 5(a-d) shows two fabricated channel designs with a channel depth of $100 \mu \mathrm{m}$ and a width of $200 \mu \mathrm{m}$ fabricated using a single pass of the laser. The performance of each device was tested by injecting dyed water into the inlet ports at a flowrate of $20 \mu \mathrm{L} \mathrm{min}^{-1}$ and observing the flow behavior. Figure 5a shows an empty Y-junction with a $15 \mathrm{~mm}$ long microchannel. Figure $5 \mathrm{~b}$ shows two liquids being pumped into the channel; the insets highlight the laminar flow that is maintained throughout the channel length. Figure 5c presents a hybrid PDMS/paper microfluidic mixer. Figure 5d depicts red and green liquids entering the serpentine microchannel with a laminar flow, each at a flow rate of $10 \mu \mathrm{L} \mathrm{min}{ }^{-1}$ and mixing together in the serpentine channel; the inset shows the resulting mixed solution at the end of the channel.

The double-sided nature of the Janus paper can be used to integrate materials compatible with many paper processing techniques on the hygroscopic/hydrophilic side. Figure 5(e-f) shows an application in which the hydrophilic side is coated with a hydrogel layer (a popular material for tissue engineering applications in which the hydrogel is seeded with live cells kept alive by providing nutrients and disposing the waste). Here, a modified device with laser-defined holes on the paper is used to deliver liquid to the hydrogel-coated hygroscopic side. The gel used for this experiment is a yellow-dyed 1\% (w/v) agarose gel, which adheres to the surface without any additional treatment. When two liquids are pumped into the PDMS-paper serpentine channel, they mix together and subsequently diffuse into the gel through small laser defined openings on the paper (denoted in the inset of Figure 5f ). The direct control and delivery of liquids on such a low-cost platform can be adapted for use in cell culturing and tissue engineering procedures, making PDMS-Janus paper structure an inexpensive but practical microfluidic platform.

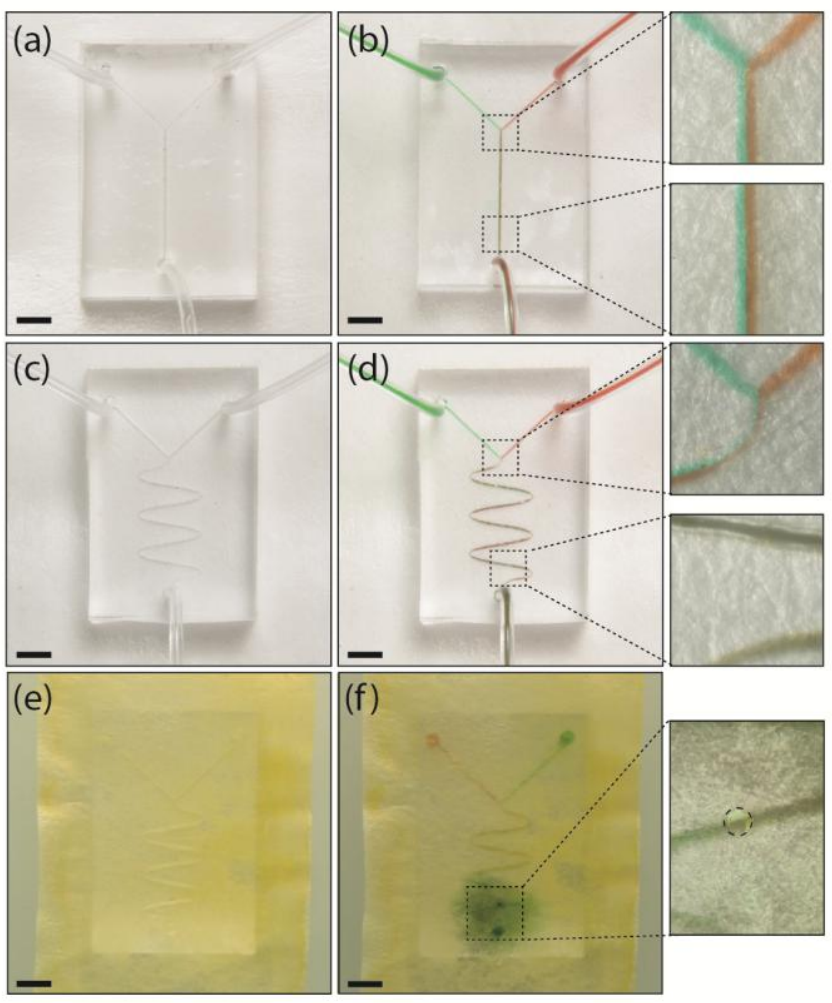

Figure 5: Various microfluidic devices fabricated using PDMS and freezer paper. All channels have a Gaussian cross-section $(200 \mu \mathrm{m}$ width and $100 \mu \mathrm{m}$ depth). (a) An empty Y-junction connected to a straight channel imaged from the PDMS side and $(b)$ with liquid flowing; the insets show a 10X magnified view of the laminar flow. (c) An empty serpentine channel imaged from the PDMS side and (d) with two dyes flowing and mixing together; the insets (10X magnification) highlight the mixing. (e) A serpentine channel imaged from the paper side, with a yellow-colored gel coating the hydroscopic face of the paper. (f) Two dyes mixing in the serpentine channel exiting the channel though a 250 pm-diameter hole in the paper (shown in the inset) and diffusing into the gel. All scale bars: $2 \mathrm{~mm}$.

\section{CONCLUSIONS}

We successfully implemented several microfluidic devices on a commercially available Janus paper (freezer paper) with one hydrophobic face and a hygroscopic one. By corona treating the hydrophobic side and the PDMS, the two were bonded irreversibly. We optimized the corona discharge duration, annealing time, and annealing temperature to achieve a strong bond. Furthermore, the hygroscopic side of the paper offers a surface morphology ideal for depositing hydrogel layers, thus enabling its use for cell culture and tissue engineering applications.

\section{ACKNOWLEDGEMENTS}

Funding for this work was provided by the National Science Foundation under grant EFRI-BioFlex \#1240443.

\section{REFERENCES}

[1] A. Lee, "The third decade of microfluidics.," Lab Chip, vol. 13, no. 9, pp. 1660-1, May 2013.

[2] X. Li, D. R. Ballerini, and W. Shen, "A perspective on paper- 
based microfluidics: Current status and future trends.," Biomicrofluidics, vol. 6, no. 1, pp. 11301-1130113, Mar. 2012.

[3] D. D. Liana, B. Raguse, J. J. Gooding, and E. Chow, "Recent advances in paper-based sensors.," Sensors (Basel)., vol. 12, no. 9, pp. 11505-26, Jan. 2012.

[4] F. Yue, T. S. Ngin, and G. Hailin, "A novel paper $\mathrm{pH}$ sensor based on polypyrrole," Sensors Actuators B Chem., vol. 32, no. 1, pp. 33-39, Apr. 1996.

[5] R. Rahimi, G. Chitnis, P. Mostafalu, M. Ochoa, S. Sonkusale, and B. Ziaie, "A low-cost oxygen sensor on paper for monitoring wound oxygenation," in The 7th International Conference on Microtechnologies in Medicine and Biology, 2013.

[6] H. Liu and R. M. Crooks, "Paper-based electrochemical sensing platform with integral battery and electrochromic read-out.," Anal. Chem., vol. 84, no. 5, pp. 2528-32, Mar. 2012.

[7] A. W. Martinez, S. T. Phillips, M. J. Butte, and G. M. Whitesides, "Patterned paper as a platform for inexpensive, low-volume, portable bioassays.," Angew. Chem. Int. Ed. Engl., vol. 46, no. 8, pp. 1318-1320, Jan. 2007.

[8] M. Reches, K. a Mirica, R. Dasgupta, M. D. Dickey, M. J. Butte, and G. M. Whitesides, "Thread as a matrix for biomedical assays.," ACS Appl. Mater. Interfaces, vol. 2, no. 6, pp. 1722-8, Jun. 2010.

[9] W. Dungchai, O. Chailapakul, and C. S. Henry, "A low-cost, simple, and rapid fabrication method for paper-based microfluidics using wax screen-printing.," Analyst, vol. 136, no. 1, pp. 77-82, Jan. 2011.

[10] G. Chitnis, Z. Ding, C.-L. Chang, C. a Savran, and B. Ziaie, "Laser-treated hydrophobic paper: an inexpensive microfluidic platform.," Lab Chip, vol. 11, no. 6, pp. 1161-5, Mar. 2011.

[11] G. Chitnis, T. Tan, and B. Ziaie, "Laser-assisted fabrication of batteries on wax paper," J. Micromechanics Microengineering, vol. 23, no. 11, p. 114016(5pp), Nov. 2013.

[12] B. Ziaie, A. Baldi, M. Lei, Y. Gu, and R. A. Siegel, "Hard and soft micromachining for BioMEMS: review of techniques and examples of applications in microfluidics and drug delivery," Adv. Drug Deliv. Rev., vol. 56, no. 2, pp. 145-172, Feb. 2004.

[13] A. Folch, Introduction to BioMEMS, 1st ed. Boca Raton, FL: CRC Press, 2012.

[14] A. C. R. Grayson, R. S. Shawgo, A. M. Johnson, N. T. Flynn, Y. Li, M. J. Cima, and R. Langer, "A BioMEMS Review: MEMS Technology for Physiologically Integrated Devices," Proc. IEEE, vol. 92, no. 1, pp. 6-21, Jan. 2004.

[15] J. Zhou, A. V. Ellis, and N. H. Voelcker, "Recent developments in PDMS surface modification for microfluidic devices.," Electrophoresis, vol. 31, no. 1, pp. 2-16, Jan. 2010.

[16] Y. Xia and G. M. Whitesides, "Soft Lithography," Angew. Chemie Int. Ed., vol. 37, no. 5, pp. 550-575, Mar. 1998.

[17] L.-M. Fu, W.-J. Ju, R.-J. Yang, and Y.-N. Wang, "Rapid prototyping of glass-based microfluidic chips utilizing twopass defocused CO2 laser beam method," Microfluid. Nanofluidics, vol. 14, no. 3-4, pp. 479-487, Oct. 2012.

[18] P.-A. Vidi, T. Maleki, M. Ochoa, L. Wang, S. M. Clark, J. F. Leary, and S. A. Lelièvre, "Disease-on-a-Chip: Mimicry of Tumor Growth in Mammary Ducts," Lab Chip, 2013.

[19] M. Lehocký, H. Drnovská, B. Lapčíková, a. . BarrosTimmons, T. Trindade, M. Zembala, and L. Lapčík, "Plasma surface modification of polyethylene," Colloids Surfaces A Physicochem. Eng. Asp., vol. 222, no. 1-3, pp. 125-131, Jul.
2003.

[20] P. Slepička, a. Vasina, Z. Kolská, T. Luxbacher, P. Malinský, a. Macková, and V. Švorčík, "Argon plasma irradiation of polypropylene," Nucl. Instruments Methods Phys. Res. Sect. B Beam Interact. with Mater. Atoms, vol. 268, no. 11-12, pp. 2111-2114, Jun. 2010.

[21] J. Kim, R. Surapaneni, and B. K. Gale, "Rapid prototyping of microfluidic systems using a PDMS/polymer tape composite.," Lab Chip, vol. 9, no. 9, pp. 1290-3, May 2009.

[22] C. S. Thompson and A. R. Abate, "Adhesive-based bonding technique for PDMS microfluidic devices.," Lab Chip, vol. 13, no. 4, pp. 632-5, Feb. 2013.

\section{CONTACT}

*B.Ziaie, tel:+1-765-404-0726; bziaie@ purdue.edu 